\title{
VASCULO-FIBROUS ARCHITECTURE OF THE MAGISTRAL PORTAL COMPLEXES
}

\author{
Ilia Chanukvadze \\ Department of General Surgery, Tbilisi State Medical University, Tbilisi, Republic of Georgia
}

\begin{abstract}
The aim of the study was the identification of anatomical characteristics of lympho-biliary interactions and their presumed connections in the intrahepatic portal tracts. Morphological investigation of vascular-fibrous structures in the intrahepatic portal tracts of 75 adults liver samples was performed. It was described that the single segments of the intrahepatic structures and their fibrous tunics constitute of three vascular-fibrous sheathes orbiculary surrounding each other. The extramural biliary mucous glands provide the connections between the lymphatic and intrahepatic biliary systems.
\end{abstract}

Keywords: lympho-biliary connections, hepatic vascular-fibrous structures

\section{INTRODUCTION}

It often is necessary to orientate on separate elements of the portal complexes and surrounding fibrous elements in human liver and biliary tract surgery (Kornev et al, 1994; Counaud, 1957). It's especially important in precision operations on liver that requires detailed notions of portal tract structure.

There cannot be the doubt that lympho-biliary connections exist at the level of hepatic profound structures (Briskin et al, 1986; Dumpe et al, 1983; Snegirevskaia et al, 1986; Bettezzati M., 1975). Causal relationship between increased intraductal pressure in cholestasis and structural changes of intercellular connections is also undisputable (Virenkov et al, 1971). Biliary hypertension causes increased permeability and transition of blocked bile into the lymphatic flow via the perisinusoidal spaces of Disse (Ostroverkhov et al, 1972). However, the spaces of Disse aren't lined with endothelium and don't belong to the lymphatic system (Alekseev, 1983; Virenkov and Kataev, 1983). We were interested in probability of any other way of blocked bile transition into the lymphatic system, namely within the bounds of the intrahepatic portal tracts.

We could not find any investigations for the possible lympho-biliary connections in the intrahepatic portal complexes in the accessible literature. How- ever, in our early works we detected an extramural location of the conduits of the biliary mucous glands into the loose connective tissue which is penetrated with lymphatic fissures and vessels.

The goal of the study was the investigation of vascular-fibrous structure of portal complexes and the detection of presumed lympho-biliary connections in those complexes.

\section{MATERIALS AND METHODS}

Investigations of 57 cadaver livers from adults of both sexes were performed. We studied the sections of the extrahepatic and large intrahepatic portal tracts prepared under the stereoscopic microscope with further reconstructions of sketched images. We studied total histotopograhic sections of the intrahepatic portal tracts prepared from celluloid blocs and stained by Van Gison, Veinhert, Hart and picrocarmine. Histological specimens were stained by Van Gison, hematoxiline and eosin, resorcine-fucsin, resorcine-picrocarmine and mucicarmine. The biliary mucous glands and lymph vessels were delineated by means of ink and gelatin mixture injection into the common bile duct. The injection was performed at a high pressure (around $300 \mathrm{~mm} \mathrm{H}_{2} \mathrm{O}$ ). The mixture flow into the lymphatic vessels was observed in each case. At the same time we studied 18 liver corrosion casts 
injected with latex beforehand within the common bile duct to investigate the surface of the biliary mucous glands.

\section{RESULTS}

The study allowed us to imagine interactions among the separate elements of the portal complexes and their connective tissue tunics as a system of three vascular-fibrous sheathes orbiculary surrounding each other. The portal vein with its fibrous sheath was the central element in a portal complex, connected with the adventitia via the collagen fibers of different directions. The central fibrous sheath formed a bed for the vein (sheath 1) (Fig. 1, 2,3 ). Solitary lymphatic vessels were distributed among the fibrous fissures of a connective tissue.

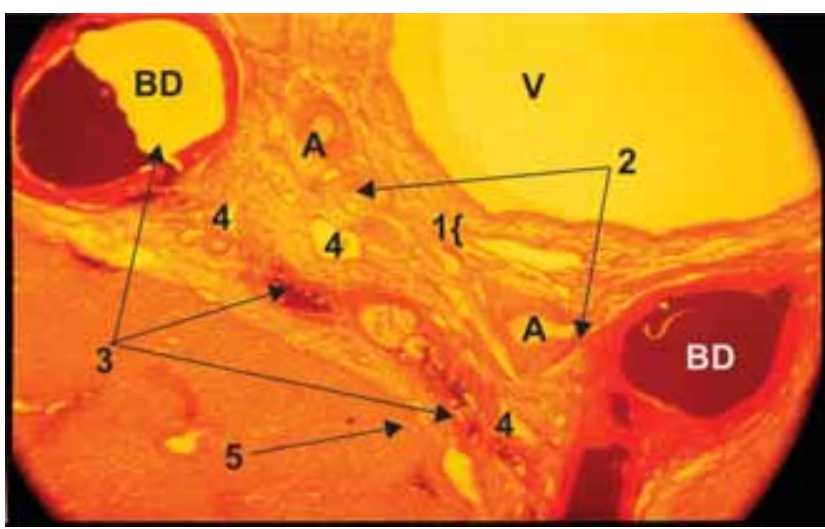

FIGURE 1. Histotopograph of portal tract

V-portal vein, A-hepatic arterial branches, B.D - bile ducts filled with ink-gelatinous mixture

1 - Fibrous sheath of portal vein, 2 - periarterial connective tissue and its lateral processes, 3 - biliary mucous glands filled with ink-gelatinous mixture, 4 - lymph vessels, 5 - perivascular fibrous capsule (Van Gison staining, $X 22)$.

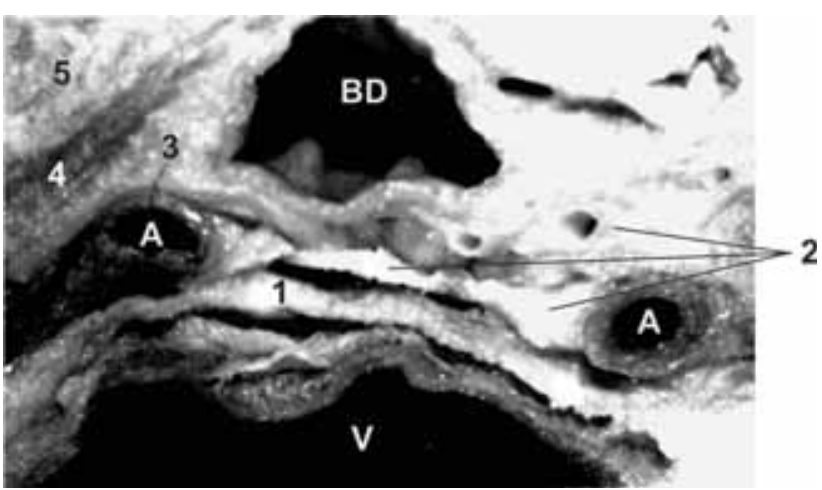

FIGURE 2. Portal tracts prepared under microscope.

$V$ - portal vein, $A$ - hepatic arterial branches, B.D - bile duct 1 - Fibrous sheath of portal vein, 2 - periarterial connective tissue and its lateral processes forming intermediate fibrous sheath between the portal vein and the bile duct, 3 - peribiliary connective tissue, 4 perivascular fibrous capsule, 5 - liver parenchima (X 16).

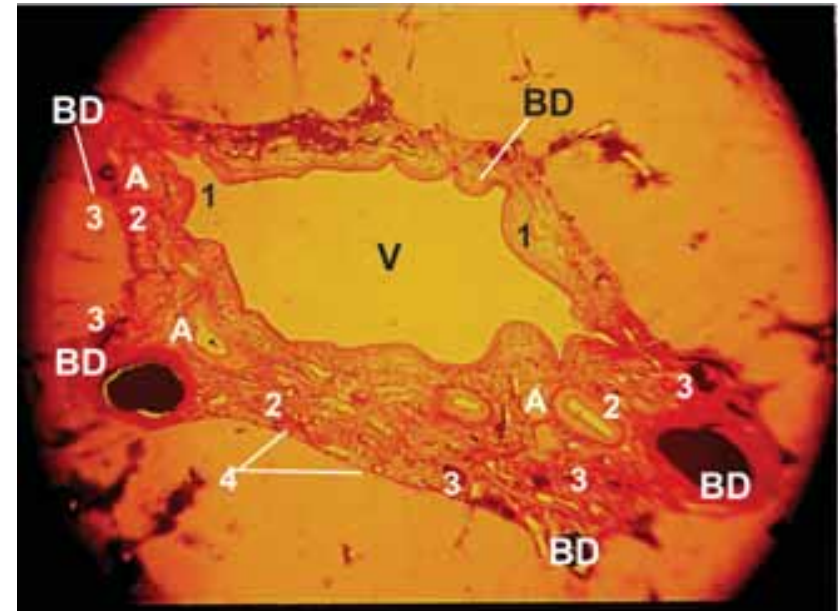

FIGURE 3. Histotopograph of total portal tract $V$ - portal vein, A - hepatic arterial branches, B.D - bile ducts filled with ink-gelatinous mixture

1 - Fibrous sheath of portal vein, 2 - periarterial connective tissue, 3 - peribiliary connective tissue and the outlines of extramural portions of biliary mucous glands, 4 - perivascular fibrous capsule (Van Gison and picrocarmine staining, $X 10$ ).

A main branch of a hepatic artery ran along the portal vein from the different sides depending on their location in a hepatic segment or sector (C. Counaud, 1957). Thus, thinner branches of the vein together with the main branch surrounded the portal vein from all sides (Fig. 3). The hepatic arterial branches had their proper fibrous sheathes in addition to the adventitia (Fig.1,2). The fibrous sheathes of the hepatic arterial branches became looser according to the further ramification of the artery (Fig. 3). The proper fibrous capsules of the sectoral and large segmental branches were linked with each other via the lateral interarterial processes formed mainly of collagenic fibres which were directed longitudinally. The arterial fibrous beds and

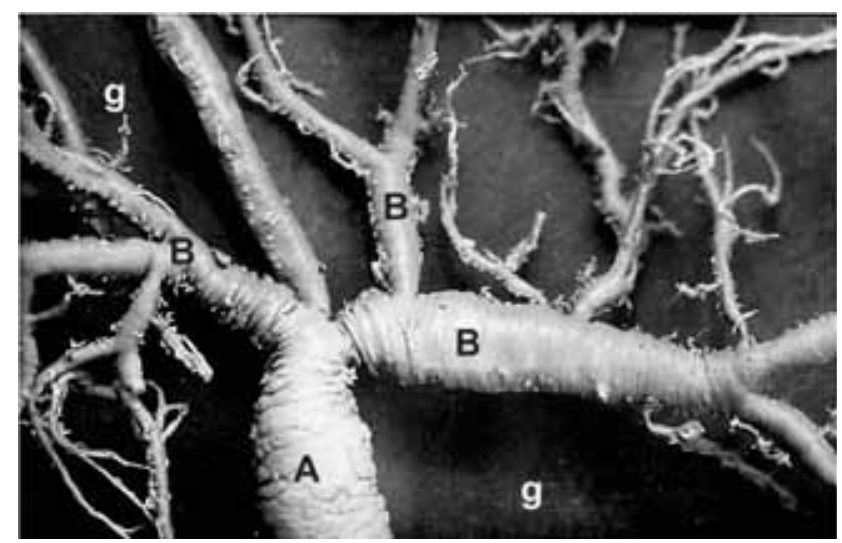

FIGURE 4. Biliary corrosion casts

A - Common bile duct with the glandular replica $(\mathrm{g})$ of various sizes distributed almost on the whole surface of the duct wall, B - intrahepatic bile ducts with the replica of biliary mucous glands $(g)$ distributed on the lateral edges (X 8). 
their lateral processes were linked with each other and with periarterial fibrous elements forming a single vascular-fibrous sheath (sheath 2 ), which irregularly enclosed vascular-fibrous structures of the portal vein and was located in the space among the vein and bile ducts (Fig. 1, 2, 3).

The bile ducts in the sectoral and segmental portal complexes occupied the most peripheral areas and quantitatively predominated over the branches of the portal vein (Fig. 3). The ducts were surrounded with the most compact fibrous structures of different orientation which formed quite large processes at the lateral edges (Fig. 2). Special attention was drawn to the histotopography of the mucous glands due to their very specific distribution

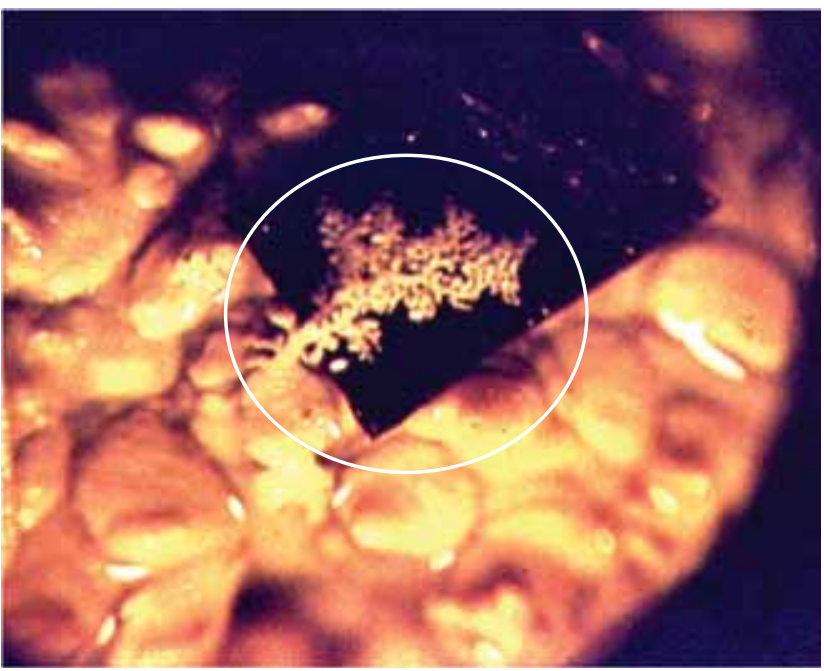

FIGURE 5. Corrosion casts of human intrahepatic bile duct. Acinar biliary glands (latex, X 12).

Replica of biliary mucous glands of different forms are concentrated at the lateral parts of the ducts (a). Long (extramural) glands form anastomoses with each other (b).

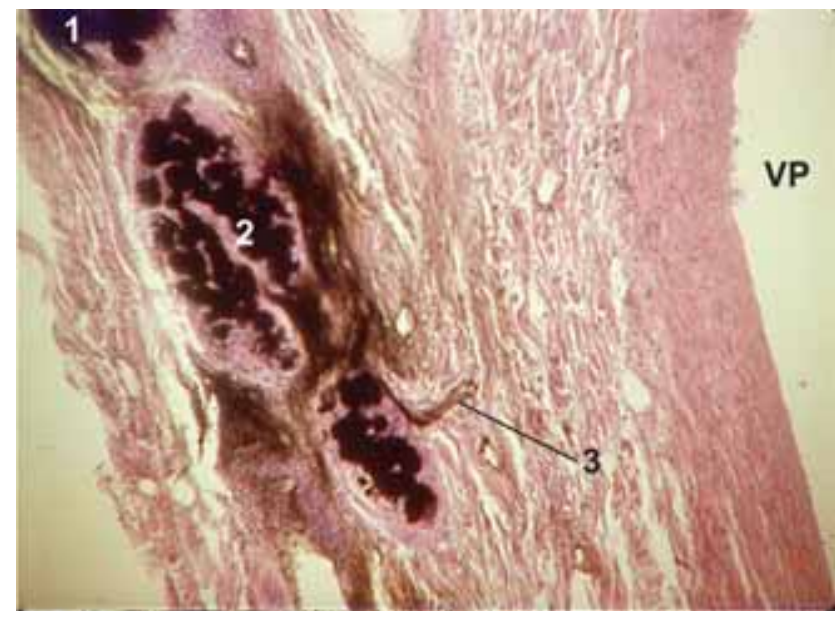

FIGURE 6. Portal tract segment

1 - Bile duct, 2 - extramural portion of biliary mucous gland injected with ink-gelatinous mixture; 3 -lymph vessel injected with ink-gelatinous mixture (H\& E, X 24). in the bile ducts (Fig. 1). An extramural distribution of the glands in the duct wall was noted in our early studies. The glandular bodies and their blind endings were located out of the duct wall and intended to extend to a considerable distance from the external layer of the duct wall (Fig. 5, 1) contrary to other gastrointestinal mucous glands of intramural location (Kornev et al, 1994; Konstantinov et al, 2000). Some of the long forms of the mucous glands penetrated the paravasal fibrous capsule reaching the portal vein capsule (Fig. 6). The parabiliary connective tissue and its lateral processes served for a bed to the biliary mucous glands and were tightly linked with the perivascular fibrous capsule, forming a fibrous frame for the glands that made an external secretory-fibrous layer of a portal complex more compact (Fig. 1, 2, 3).

All the elements of a portal complex (the portal vein, the hepatic arterial branches, the bile ducts, the mucous glands, the nerves, the lymphatic vessels and their surrounding fibrous structures) were surrounded with the common perivascular fibrous capsule (PNA) which was connected tightly with parabiliary tissues via the interweaved collagenic and elastic bundles (Fig. 2). These connections were so firm that it was impossible to separate them even when preparing under the microscope. That's why we consider both the parabiliary connective tissue and the perivascular capsule to be a single external fibrous sheath (sheath 3 ) for all the vascular-secretory and fibrous structures of the portal complex.

The highest concentration of the lymphatic capillaries and vessels was observed in the depth of the parabiliary connective tissue and at their lateral processes which contained the extramural portions of the biliary mucous glands (Fig.1).

The lymphatic fissures and capillaries were connected with the biliary glands. On all the specimens, flooded with the ink-gelatinous mixture, increased permeability of the walls of the mucous glands or their anatomical impairment was observed that was followed with the leaking of the mixture into the parabiliary connective tissue, the lymphatic fissures and spaces (Fig. 6), where the transition of the ink-gelatinous mixture into the lymphatic flow was indicated.

\section{DISCUSSION}

An extramural location of biliary mucous glands at the lateral edges of the intrahepatic bile ducts, their remoteness from the duct walls and connections with other portal structures would seem to 
give a reason to consider these glands to be the independent anatomical elements and designate them as "peribiliary glands" (Terasaki, Nakanuma et al, 1997; Nakanuma et al, 1994). However, we believe this term not to reflect their true functional and anatomical belonging to bile ducts and according to Paris Nomina Anatomica we call these glands "glandula mucosae biliosae" (PNA). A histotopography of the extrahepatic and intrahepatic glands is different: an extramural position of the glands is specific to the intrahepatic bile ducts, while the extrahepatic bile glands are distributed in the duct wall circularly and usually don't expend out of its bounds (Fig. 4). It's important to distinguish the peculiarities of the biliary mucous glands in biliary tract surgery as the glands with their microcirculation system may be captured during the common bile duct or common hepatic duct suturing that may lead to the necrosis of the glands with a further insufficiency of the adaptive layers. As for the intrahepatic lobular ducts and the bile ducts of subsequent degrees, the glands are distributed extramuraly at the lateral edges, the internal and the external hemi circumferences of the ducts don't contain the glands, and it makes favourable conditions for suturing.

We don't doubt in the possibility of the lymphobiliary connections at the level of the spaces of Disse (as a compensatory mechanism in the condi-

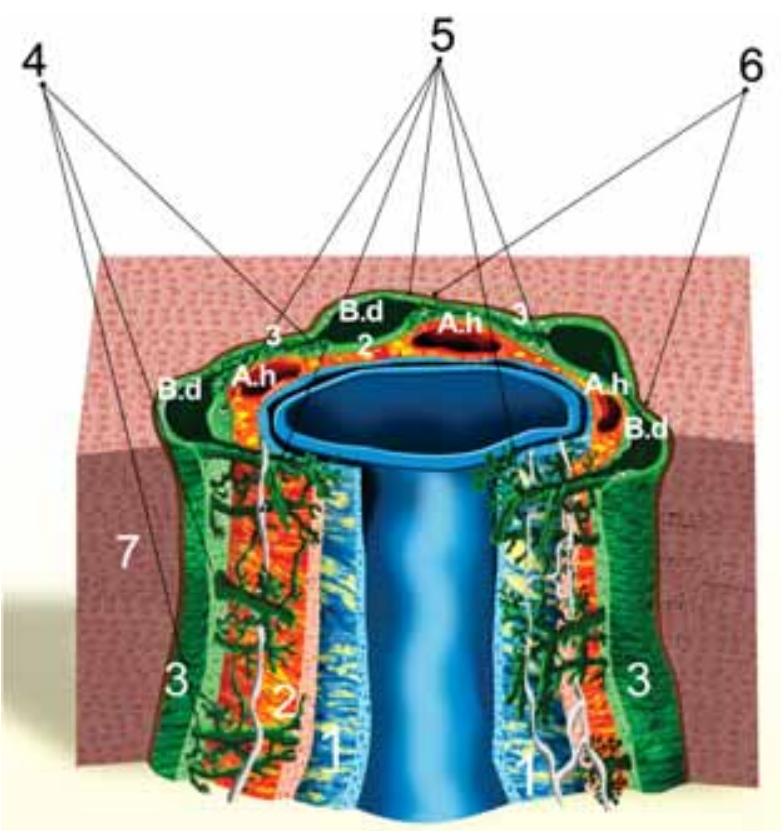

FIGURE 7. V.P. Portal vein; B.D. Bile ducts; A. Hepatic arteries. 1. Fibrous sheath of portal vein (sheath 1); 2. Fibrous interarterial sheath (sheath 2); 3. Peribiliary connective tissue and perivascular fibrous capsule (6) forming common outer fibrous capsule for the elements of the portal complex (sheath 3). 4. Biliary mucous glands; 5. Lymph vessels; 7. Liver parenchima. tions of increased intraductal pressure) (Ostroverkhov et al, 1973; Dedov, 1973). At the same time we revealed another way of forming lympho-biliary fistulas, namely, in the peribiliary connective tissue via the extramural portions of the biliary mucous glands. On the basis of these findings intrahepatic portal tracts may be considered as one of the possible areas of the lympho-biliary connections where the blocked bile floods into the lymphatic system.

Studying the interactions among the elements of lobular, sectoral and large segmental portal complexes, various authors has reported the distribution of the hepatic arterial branches and the bile ducts on the different surfaces of the portal vein, which is known as a main landmark of the portal triad (Dumpe et al, 1983; Zatolokin, 1974; Ostroverkhov and Zabrodskaia, 1972; Shapkin et al, 1983; Counaud, 1957, etc). Those works describing vascular architecture of the liver are based on the study of the corrosion casts of the portal vein, the hepatic artery and the bile ducts, but the surrounding fine structures don't remain on the casts, that don't allow studying interactions among all of the stromal and vascular elements of the portal tract. An introduction of the histotopographic methods made it possible (Kovanov et al, 1967; Kovanov et al, 1973, etc). However, there's a few works suggested which use these methods to study the fibrous structures of intrahepatic portal complexes (Pavlinov, 1969). Despite B.G Pavilinov demonstrated the presence of the fibrous capsules around the portal vein and the hepatic arterial branches as long ago as 1969 and the investigations of other soviet scientists confirmed those results (Thoidze and Chanukvadze, 1978; Razumnaia, 1979; Chanukvadze, 1988), a largly scientific society has left them without any serious attention. Thus, the presence of formed fibrous structures each of the portal triad elements hasn't been reflected in the publications dealing with the problems of hepatology (Ostroverkhov and Zabrodskaia, 1972; Fegereshanu et al, 1976; Terasaki et al, 1997).

The spatial and histotopographical interactions among the blood vessels of various calibers, bile ducts and their mucous glands in the system of the intrahepatic fibrous frame of portal complexes described in the article are novel. The results of the investigations allow us taking a novel view of the system of the portal complexes, imagining as a scheme (Fig. 7) of the circulatory distributed vascular-fibrous layers reflecting the peculiarities of the lobular, sectoral and large segmental portal tracts, helping surgeons orientate on the separate elements of the portal triad in various surgical procedures, especially in microsurgery. 


\section{REFERENCES}

1. Alekseev B.V. [Surgery of Thoracic Duct]. $4^{\text {th }}$ ed.1983: 92-113

2. Briskin B.S. et al [Errors and Complications in Lymphosorption]. Khirurgiya 1986; 12: 109-104

3. Virenkov Y.A., Kataev S.M. [Hepatic Microcirculatory Flow According to the Evidences of Electronic Microscopy]. Arkhiv Anatomii, Gistologii, Embriologii 1983, 4:21-24

4. Virenkov Y.A, Makarenko E.P., Martirosova M.T. [Hepatic Lymphocirculation in Biliary Hypertension]. Khirurgiya 1971, 2: 35-40

5. Gugushvili L.L. [Hepatic Retrograde Blood Circulation and Portal Hypertension]. Moscow: "Meditsina" , 1972:196

6. Dumpe E.P., Krilov L.B., Shurkalin B.K. [Thoracic Duct Drainage and Lymphosorption in Complex Management of Obstructive Jaundice and Pancreonecrosis. Managed Lymphodrainage in Clinical Practice and Experimants]. Moscow 1983: 35-39.

7. Zatolokin V.D. [Anatomical Resections of Hepatic Lobe]. Abstract of Dissertation for M.D., Moscow, 1974

8. Kovanov V.V., Anikina T.I. [Surgical Anatomy of Human Fasciae and Tissue Spaces]. $2^{\text {nd }}$ ed., Moscow, "Meditsina", 1967: 428

9. Kovanov V.V., Anikina T.I., Razumnaia T.A. [Surgical Significance of the Connective Tissue Structures of the Posterior Part of Human Liver]. [Scientific Methodological Problems of Soft Tissue Studying.] Gorkyi, Kirov Medical Institute,1973:137-146.

10. Kornev O.S., Davlatkindev F.A., Kim B.Kh. [Thoracic Duct Measuring Drainage in Urgent Surgery]. Khirurgiya 3:90-93.

11. Konstantinov B.A., Gotie S.V., TsirukInikova O.M., Filin A.V. [Living Related Liver Transplantation. The First Clinical Experience]. Khirurgiya 2000; 3: 4-9

12. Ostroverchov G.S., Zabrodskaiya V.F [Surgical Anatomy of Liver and Biliary Tract]. [Abdominal Surgical Anatomy]. Leningrad, "Meditsina", 1972: 297-384

13. Ostroverchov G.S., Zabrodskaiya V.F., Aliev A.Z. [Compensatory Role of Lymphatic System in Choledochal and Cystic Duct Obstruction in Experiment]. [The Works of M.M.I. 2]. $1^{\text {th }}$ ed., Vol. 18: 112-117

14. Snegirevskaiya E.S., Veselov V.S. [Changes in Plasmogenic Hepatocytes and their Specific Sites of Intercellular Junctions in Complicated Forms of Cholelithiasis]. Arkhiv Anatomii, Gistologii, Embriologii. 1986; 1: 12-15.

15. Thoidze Sh.S., Chanukvadze I.M. [Interactions Among Liver Paravascular Fibrouse Structures]. [Matherials of Transcaucasian Conference of Morphologists]. Baku, 1978: 268-369.
16. Fegereshanu I., lonesku-Budjor K., Aloman D., Albu A. [Surgery of Liver and Intrahepatic Biliary Tracts]. Bucharest, 1976: 537

17. Pavlinov B.G. [Structure and Development of Fibrous Tissue around Hepatic Blood Vessels and Bile Ducts]. Dissertation for M.D., Omsk Medical State Institute; 1969: 148.

18. Razumnaiya T.A. [Development and Structure of Soft Tissue of Human Liver and Its Significance in Surgery]. Dissertation for M.D., Moscow Sechenov Medical Institute 1, 1976: 36.

19. Razumnaiya T.A. [Morphological and Topographical Peculiarities of Fixative System of Human Liver]. [Clinical Aspects of Surgical Anatomy in Experimental Surgery]. Moscow Sechenov Medical Institute 1, 1979:102-105.

20. Chanukvadze I.M. [Structure and Connections of Paravasal Connective Tissue in Liver]. Dissertation for M.D.Tbilisi Medical Institute; 1979

21. Chanukvadze I.M. [Structure and Connections of Fibrous Sheathes of Portal Complexes and Hepatic Veins. Surgical Anatomy and Experimental Morphology of Liver]. [Collection of Scientific Works of Tbilisi State Medical Institute]. Tbilisi, T.S.M.I.; 1989: 13-33.

22. Shapkin V.S., Thoidze Sh.S., Izraelishvili M.Sh [Surgical Procedures on Liver in the Conditions of Extracorporal Blood Circulation]. Tbilisi, "Sabchota Sakartvelo"; 1983: 108.

23. Sherlock Sh., Dooli G. [Deseases of Liver and Biliary Tracts]. Moscow, "Meditsina"; 1999: 859.

24. Dedo D.D. Canula for Maintaining Long-term Thoracic Duct Fistulas. Surgery, 1973; vol. 73, 5:791-793.

25. Bettezzati M. La Dekompression del Sistema Lymphatic in Corso di Hipertensione Portall. Minerva Chir., 1975; vol. 30, 7: 383-384.

26. Bartos V., Brezek V. [Significance of Thoracic Duct Drinage in Clinical Medicine]. Chirurgy, 1973; 4:110-114

27. Counaud C. Le Foie: Etudes Anatomiques et Chirurgikales. Masson, Paris; 1957

28. Tanaka K., Uemato S., Tokunoga Y. et al. Surgical Techniques and Innovation on Living Related Liver Transplantation. Ann Surg 1997, vol. 217, 1: 82-91

29. Terasaki S., Nakanuma Y., Unoura M et al. Involvment of Peribiliary Glands in Primary Sclerosing Cholangitis: Histopathologic Study. Internal Medicine 1997, vol.36, 11:766-770

30. Nakanuma Y., Sasaki M., Terada T., Kenichi H. Intrahepatic Peribiliary Glands of Human.II. Pathological Spectrum. J Gastroenterol Hepatol, 1994, 9:80-86. 